\title{
LIAPUNOV FUNCTIONALS FOR SMOLUCHOWSKI'S COAGULATION EQUATION AND CONVERGENCE TO SELF-SIMILARITY
}

\author{
Philippe Laurençot ${ }^{1}$ and Stéphane Mischler ${ }^{2}$
}

July 6, 2004

\begin{abstract}
An alternative proof of the convergence to self-similar profiles for solutions to the Smoluchowski coagulation equation with constant coagulation kernel is provided. In contrast to the previous approaches which rely on the Laplace transform, a dynamical systems approach is used on the equation written in self-similar variables, for which several Liapunov functionals are identified.
\end{abstract}

Keywords. Coagulation, self-similar solution, Liapunov functional

AMS Subject Classification. 45K05, 45M05, 37L45, 82C21

\section{Introduction}

The Smoluchowski coagulation equation is a mean-field model for the dynamics of a system of particles growing by successive mergers [7, 23, 24]. Assuming that each particle is fully identified by its volume, the Smoluchowski coagulation equation provides a description of the evolution of the volume distribution function $f=f(t, y) \geq 0$ as a function of the time $t \geq 0$ and the volume $y \in \mathbb{R}_{+}:=(0,+\infty)$ and reads

$$
\partial_{t} f(t, y)=\frac{1}{2} \int_{0}^{y} a\left(y-y^{\prime}, y^{\prime}\right) f\left(t, y-y^{\prime}\right) f\left(t, y^{\prime}\right) d y^{\prime}-\int_{0}^{\infty} a\left(y, y^{\prime}\right) f(t, y) f\left(t, y^{\prime}\right) d y^{\prime}
$$

for $(t, y) \in(0,+\infty) \times \mathbb{R}_{+}$. Here, the function $a$ is the coagulation kernel and satisfies $a\left(y, y^{\prime}\right)=a\left(y^{\prime}, y\right) \geq 0$ for $\left(y, y^{\prime}\right) \in \mathbb{R}_{+}^{2}$.

For homogeneous coagulation kernels satisfying $a\left(\xi y, \xi y^{\prime}\right)=\xi^{\lambda} a\left(y, y^{\prime}\right)$ for some $\lambda \leq 1$, it is conjectured by physicists that the distribution function $f$ behaves in a self-similar way for large times. More precisely, the dynamical scaling hypothesis asserts that

$$
f(t, y) \sim f_{S}(t, y)=\frac{1}{s(t)^{2}} g_{S}\left(\frac{y}{s(t)}\right)
$$

\footnotetext{
${ }^{1}$ Mathématiques pour l'Industrie et la Physique, CNRS UMR 5640, Université Paul Sabatier - Toulouse 3 , 118 route de Narbonne, F-31062 Toulouse cedex 4, France. E-mail: laurenco@mip.ups-tlse.fr

${ }^{2}$ CEREMADE, CNRS UMR 7534, Université Paris IX-Dauphine, Place du maréchal De Lattre de Tassigny, F-75775 Paris cedex 16, France. E-mail: mischler@ceremade.dauphine.fr
} 
after a sufficiently large time, and $f_{S}$ is a self-similar solution to the coagulation equation, see $[6,19]$ and the references therein. Here, the particle mean volume $s(t)$ and the profile $g_{S}$ are to be determined and depend on the coagulation kernel $a$ but not on the "details" of the initial data. Several computational studies have been performed to check the dynamical scaling hypothesis (1) and seem to support its validity [10, 12, 14, 20]. Also, rather precise information on the profile $g_{S}$ have been obtained by formal arguments in $[6,19]$. From the rigorous point of view, the validity of (1) is still an open problem, except for the constant kernel $a\left(y, y^{\prime}\right)=1$ and the additive kernel $a\left(y, y^{\prime}\right)=y+y^{\prime}[2,4,5,13,21,22]$. In fact, for other homogeneous coagulation kernels, the first difficulty encountered is the existence of the scaling profile $g_{S}$ which satisfies a nonlinear and nonlocal integro-differential equation. For a wide class of homogeneous coagulation kernels, such an existence result has been recently obtained in $[9,11]$. Still, the uniqueness of the profile (in a suitable class) and the convergence to self-similar solutions are open problems.

For the constant and additive kernels, explicit formulae for $g_{S}$ are available [6]. More precisely, when $a\left(y, y^{\prime}\right)=1$, the function

$$
f_{m}(t, y):=\frac{1}{t^{2}} g_{m}\left(\frac{y}{t}\right), \quad g_{m}(y):=\frac{4}{m} \exp \left(-\frac{2 y}{m}\right)
$$

is a self-similar solution to the Smoluchowski coagulation equation for any $m \geq 0$ and satisfies

$$
\int_{0}^{\infty} y f_{m}(t, y) d y=\int_{0}^{\infty} y g_{m}(y) d y=m
$$

for each $t \geq 0$.

For the constant kernel, the dynamical scaling hypothesis $(1)$ becomes $f(t) \sim f_{\varrho}(t)$ for large times, where $\varrho$ denotes the first moment of the initial datum $f(0)$. It has been proved rigorously in $[4,13]$ for rapidly decaying initial data, the convergence being uniform on compact subsets of $\mathbb{R}_{+}$. Different proofs have been subsequently supplied by a probabilistic approach in $[2,5]$ but the convergence only takes place in the weak topology of $L^{1}\left(\mathbb{R}_{+}\right)$. More recently, other self-similar solutions to the Smoluchowski coagulation equation with constant kernel have been identified in [22], and their domains of attraction for the weak convergence of measures have been characterized. Unlike (2), these self-similar solutions have an infinite first moment when $a\left(y, y^{\prime}\right)=1$. In addition, a class of initial data for which the convergence to self-similarity takes place in $L^{\infty}$ is identified in [21], thereby extending and improving the previous results. Similar results are available for the additive kernel [3, 5, 21, 22].

A common feature of the above mentioned works is that the proof of (1) always relies on the use of the Laplace transform. Our aim in this work is to show that an alternative approach can be used for the constant kernel. In fact, the main achievement of this work is the construction of Liapunov functionals for the Smoluchowski coagulation equation with constant kernel written in self-similar variables (Section 2). One of the main tools which we used here is several adaptations of an inequality established by Aizenman \& Bak [1]. The convergence to self-similarity for the weak topology of $L^{1}\left(\mathbb{R}_{+}\right)$will then follow (Section 3). 
For $k \in \mathbb{R}$, we set $L_{k}^{1}\left(\mathbb{R}_{+}\right):=L^{1}\left(\mathbb{R}_{+},\left(1+y^{k}\right) d y\right)$ and

$$
M_{k}(f):=\int_{0}^{\infty} y^{k} f(y) d y \text {. }
$$

We consider an initial datum $f^{\text {in }}$ satisfying

$$
f^{\text {in }} \in \mathcal{Y}_{p}:=\left\{u \in L_{2}^{1}\left(\mathbb{R}_{+}\right), u \geq 0 \text { such that } u^{p} \in L_{1}^{1}\left(\mathbb{R}_{+}\right)\right\},
$$

where $p \in(1, \infty)$. We denote by $f$ the corresponding solution to the Smoluchowski coagulation equation with constant kernel, that is, the solution to

$$
\begin{aligned}
\partial_{t} f & =Q_{c}(f), \quad(t, y) \in \mathbb{R}_{+}^{2}, \\
f(0) & =f^{i n}, \quad y \in \mathbb{R}_{+},
\end{aligned}
$$

where

$$
Q_{c}(f)(y):=\frac{1}{2} \int_{0}^{y} f\left(y-y^{\prime}\right) f\left(y^{\prime}\right) d y^{\prime}-\int_{0}^{\infty} f(y) f\left(y^{\prime}\right) d y^{\prime}, \quad y \in \mathbb{R}_{+} .
$$

We recall that $f$ satisfies

$$
\int_{0}^{\infty} y f(t, y) d y=\varrho:=\int_{0}^{\infty} y f^{i n}(y) d y
$$

for each $t \geq 0$, cf. $[2,17]$ and the references therein. We introduce the self-similar variables $(\ln (1+t), y /(1+t))$ and put

$$
f(t, y)=\frac{1}{(1+t)^{2}} g\left(\ln (1+t), \frac{y}{1+t}\right)
$$

for $(t, y) \in \mathbb{R}_{+}^{2}$. Then $g$ is a solution to

$$
\begin{aligned}
\partial_{t} g & =y \partial_{y} g+2 g+Q_{c}(g), \quad(t, y) \in \mathbb{R}_{+}^{2}, \\
g(0) & =f^{i n}, \quad y \in \mathbb{R}_{+} .
\end{aligned}
$$

Observe that, in terms of $g$, the dynamical scaling hypothesis $(1) \operatorname{reads} g(t) \sim g_{\varrho}$ for large times (recall that $g_{\varrho}$ is defined in (2) and $\varrho$ in (8)). Also, since $f_{\varrho}$ is a self-similar solution to $(5)$, it is clear that $g_{\varrho}$ is a stationary solution to (10). The validity of $(1)$ thus reduces to the question of convergence of $g$ towards the steady state $g_{\varrho}$.

We next introduce the functionals

$$
\begin{aligned}
& H_{0}(u \mid v):=\int_{0}^{\infty}\left\{u(y)\left(\ln \left(\frac{u(y)}{v(y)}\right)-1\right)+v(y)\right\} d y \geq 0, \\
& H_{1}(u \mid v):=\int_{0}^{\infty}\left\{u(y)\left(\ln \left(\frac{u(y)}{v(y)}\right)-1\right)+v(y)\right\} y d y \geq 0 \\
& L_{1}(u \mid v):=\int_{0}^{\infty}|u(y)-v(y)| d y \geq 0 .
\end{aligned}
$$

With these notations, our main result is the following. 
Theorem 1 The functions $H_{0}\left(g \mid g_{\varrho}\right) / M_{0}(g), H_{1}\left(g \mid g_{\varrho}\right), H_{0}\left(G \mid G_{\varrho}\right)$ and $L_{1}\left(G \mid G_{\varrho}\right)$ are nonincreasing functions of time, where

$$
G(t, y):=\int_{y}^{\infty} g\left(t, y^{\prime}\right) d y^{\prime}, \quad G_{\varrho}(y):=\int_{y}^{\infty} g_{\varrho}\left(y^{\prime}\right) d y^{\prime}=\frac{\varrho}{2} g_{\varrho}(y)
$$

for $(t, y) \in \mathbb{R}_{+}^{2}$.

The choice of the functionals $H_{0}, H_{1}$ and the proof of Theorem 1 heavily rely on the explicit formula (2) for the self-similar profile $g_{\varrho}$. Though we believe that the Liapunov functional approach could apply to a larger class of coagulation coefficients than the Laplace transform, finding Liapunov functionals for non-constant coagulation coefficients seems to be a rather difficult task.

As a consequence of Theorem 1, we obtain the following convergence result, already obtained in $[2,5,22]$ by different methods.

Theorem 2 For any $\psi \in L^{\infty}\left(\mathbb{R}_{+}\right)$, we have

$$
\lim _{t \rightarrow+\infty} \int_{0}^{\infty} g(t, y) \psi(y) d y=\int_{0}^{\infty} g_{\varrho}(y) \psi(y) d y
$$

that is, $\{g(t)\}$ converges weakly towards $g_{\varrho}$ in $L^{1}\left(\mathbb{R}_{+}\right)$as $t \rightarrow+\infty$. In other words,

$$
\lim _{t \rightarrow+\infty}(1+t) \int_{0}^{\infty} f(t, y) \psi\left(\frac{y}{(1+t)}\right) d y=\int_{0}^{\infty} g_{\varrho}(y) \psi(y) d y
$$

The convergence to self-similar solutions obtained in Theorem 2 is only with respect to the weak topology of $L^{1}\left(\mathbb{R}_{+}\right)$. Improving this convergence with the approach developed in this paper would, for instance, require to obtain uniform $L^{q}$-bounds on $g(t)$ for some $q>1$, which we have yet been unable to prove. In [21], by means of the Laplace transform, a class of initial data is identified for which convergence in $L^{\infty}\left(\mathbb{R}_{+}\right)$holds true.

Remark 3 It is likely that Theorems 1 and 2 are also valid for initial data in the space $\mathcal{Y}_{\ell}$ defined by

$$
\mathcal{Y}_{\ell}:=\left\{u \in L_{2}^{1}\left(\mathbb{R}_{+}\right), u \geq 0, \quad u \ln u \in L_{1}^{1}\left(\mathbb{R}_{+}\right)\right\} .
$$

Indeed, it is the natural space on which $H_{0}\left(g \mid g_{\varrho}\right)$ and $H_{1}\left(g \mid g_{\varrho}\right)$ are well-defined. However, the convergence of the approximation scheme outlined at the end of Section 2 (in particular, the convergence of $H_{0}$ and $H_{1}$ ) seems to be more technical to justify, but it might be possible to argue as in [16, Section 3]. 


\section{Liapunov functionals}

This section is devoted to the proof of Theorem 1. Since $H_{0}$ and $H_{1}$ involve logarithmic terms, a rigorous proof of Theorem 1 requires two steps: one first considers initial data for which the solution $g$ to (10), (11) is bounded from below by a negative exponential, for which the computations below can be done. The second step is to use the continuous dependence of the solutions to (10), (11) on the initial data to deduce by an approximation argument that Theorem 1 holds true for every $f^{i n} \in \mathcal{Y}_{p}$. To simplify the presentation, we will however only provide a formal proof of Theorem 1, and refer to the end of the section for a discussion of the approximation procedure.

We first recall that the time evolution of $M_{i}(g), i=0,1,2$, can be computed explicitly.

Lemma 4 For $t \geq 0$, we have

$$
\begin{aligned}
\frac{d M_{0}(g)}{d t} & =\frac{M_{0}(g)\left(2-M_{0}(g)\right)}{2}, \\
M_{1}(g(t)) & =M_{1}\left(f^{i n}\right)=\varrho \\
\frac{d M_{2}(g)}{d t} & =-M_{2}(g)+\varrho^{2},
\end{aligned}
$$

that is,

$$
\begin{aligned}
& M_{0}(g(t))=2+\frac{2\left(M_{0}\left(f^{i n}\right)-2\right)}{M_{0}\left(f^{i n}\right)\left(1-e^{-t}\right)+2 e^{-t}} e^{-t}, \\
& M_{2}(g(t))=e^{-t} M_{2}\left(f^{i n}\right)+\left(1-e^{-t}\right)\left(M_{1}\left(f^{i n}\right)\right)^{2} .
\end{aligned}
$$

Proof of Lemma 4. We recall that $g$ satisfies

$$
\begin{aligned}
& \int_{0}^{\infty}\left(g(t, y)-f^{i n}(y)\right) \psi(y) d y \\
= & \int_{0}^{t} \int_{0}^{\infty}\left(\psi(y)-y \partial_{y} \psi(y)\right) g(s, y) d y d s \\
+ & \frac{1}{2} \int_{0}^{t} \int_{0}^{\infty} \int_{0}^{\infty}\left(\psi\left(y+y^{\prime}\right)-\psi(y)-\psi\left(y^{\prime}\right)\right) g(s, y) g\left(s, y^{\prime}\right) d y d y^{\prime} d s
\end{aligned}
$$

for every $\psi \in W^{1, \infty}\left(\mathbb{R}_{+}\right)$and every $t \geq 0$. We take $\psi \equiv 1$ in $(20)$ and obtain that

$$
M_{0}(g(t))=M_{0}\left(f^{i n}\right)+\frac{1}{2} \int_{0}^{t} M_{0}(g(s))\left(2-M_{0}(g(s))\right) d s,
$$

from which (17) readily follows. The formulae (18) and (19) also formally follow from (20) with $\psi(y)=y$ and $\psi(y)=y^{2}$, respectively. Since these functions do not belong to $W^{1, \infty}$, an approximation argument has to be used, first with $\psi(y)=\min \{y, R\}$ and then with $\psi(y)=y \min \{y, R\}$. The formulae for $M_{1}(g)$ and $M_{2}(g)$ are then obtained by letting $R \rightarrow+\infty$. 
Proposition 5 For $t_{2} \geq t_{1} \geq 0$, we have

$$
\frac{H_{0}\left(g\left(t_{2}\right) \mid g_{\varrho}\right)}{M_{0}\left(g\left(t_{2}\right)\right)} \leq \frac{H_{0}\left(g\left(t_{1}\right) \mid g_{\varrho}\right)}{M_{0}\left(g\left(t_{1}\right)\right)} .
$$

The proof of Proposition 5 relies on the following observation due to Aizenman \& Bak [1, Proposition 4.3].

Lemma 6 Introducing

$$
D_{A B}(g):=\int_{0}^{\infty} \int_{0}^{\infty} g(y) g\left(y^{\prime}\right) \ln \left(\frac{g\left(y+y^{\prime}\right)}{g\left(y^{\prime}\right)}\right) d y d y^{\prime},
$$

we have

$$
D_{A B}(g)+M_{0}(g)^{2} \leq 0 .
$$

Proof of Proposition 5. We first notice that

$$
H_{0}\left(g \mid g_{\varrho}\right)=\int_{0}^{\infty} g(\ln g-1) d y+4-\ln \left(\frac{4}{\varrho}\right) M_{0}(g) \text {. }
$$

We then infer from (10), (17) and Lemma 6 that

$$
\begin{aligned}
\frac{d}{d t} H_{0}\left(g \mid g_{\varrho}\right)= & \int_{0}^{\infty} \ln g \partial_{t} g d y-\ln \left(\frac{4}{\varrho}\right) \frac{d M_{0}(g)}{d t} \\
= & \int_{0}^{\infty} g \ln g d y+M_{0}(g)+\frac{1}{2} \int_{0}^{\infty} \int_{0}^{\infty} \ln \left(g\left(y+y^{\prime}\right)\right) g(y) g\left(y^{\prime}\right) d y^{\prime} d y \\
& -M_{0}(g) \int_{0}^{\infty} g(y) \ln (g(y)) d y+\ln \left(\frac{4}{\varrho}\right) \frac{M_{0}(g)\left(M_{0}(g)-2\right)}{2} \\
= & \frac{2-M_{0}(g)}{2} H_{0}\left(g \mid g_{\varrho}\right)-\left(M_{0}(g)-2\right)^{2}+\frac{1}{2}\left(D_{A B}(g)+M_{0}(g)^{2}\right) \\
\leq & \frac{1}{M_{0}(g)} \frac{d M_{0}(g)}{d t} H_{0}\left(g \mid g_{\varrho}\right) .
\end{aligned}
$$

Integrating the above inequality over $\left(t_{1}, t_{2}\right)$ yields Proposition 5 .

Proposition 7 For $t_{2} \geq t_{1} \geq 0$, we have

$$
H_{1}\left(g\left(t_{2}\right) \mid g_{\varrho}\right)+\int_{t_{1}}^{t_{2}} D_{1}(g(s)) d s \leq H_{1}\left(g\left(t_{1}\right) \mid g_{\varrho}\right)
$$

where

$$
D_{1}(g):=\varrho \int_{0}^{\infty} \mathcal{G}\left(\frac{2}{\varrho}+\partial_{y} \ln \mathcal{G}\right)^{2} d y \geq 0
$$

and

$$
\mathcal{G}(t, y):=\int_{y}^{\infty} G\left(t, y^{\prime}\right) d y^{\prime}, \quad(t, y) \in \mathbb{R}_{+}^{2}
$$


As for Proposition 5, the cornerstone of the proof of Proposition 7 is the following lemma, which is in the spirit of [1, Proposition 4.3].

Lemma 8 We have

$$
\int_{0}^{\infty} \int_{0}^{\infty} y^{\prime} g(y) g\left(y^{\prime}\right) \ln \left(\frac{g\left(y^{\prime}\right)}{g\left(y+y^{\prime}\right)}\right) d y^{\prime} d y \geq \varrho \int_{0}^{\infty} \frac{|G(y)|^{2}}{\mathcal{G}(y)} d y .
$$

Proof of Lemma 8. We proceed along the lines of the proof of [1, Proposition 4.3]. Setting

$$
I:=\int_{0}^{\infty} \int_{0}^{\infty} y^{\prime} g(y) g\left(y^{\prime}\right) \ln \left(\frac{g\left(y^{\prime}\right)}{g\left(y+y^{\prime}\right)}\right) d y^{\prime} d y
$$

and $\varphi(r)=r \ln r, r \geq 0$, we deduce from the convexity of $\varphi$, the identity

$$
\mathcal{G}(y)=\int_{0}^{\infty} y^{\prime} g\left(y+y^{\prime}\right) d y^{\prime}, \quad y \in \mathbb{R}_{+}
$$

and the Jensen inequality that

$$
\begin{aligned}
I & =\int_{0}^{\infty} g(y) \mathcal{G}(y) \int_{0}^{\infty} \varphi\left(\frac{g\left(y^{\prime}\right)}{g\left(y+y^{\prime}\right)}\right) \frac{y^{\prime} g\left(y+y^{\prime}\right)}{\mathcal{G}(y)} d y^{\prime} d y \\
& \geq \int_{0}^{\infty} g(y) \mathcal{G}(y) \varphi\left(\int_{0}^{\infty} \frac{g\left(y^{\prime}\right)}{g\left(y+y^{\prime}\right)} \frac{y^{\prime} g\left(y+y^{\prime}\right)}{\mathcal{G}(y)} d y^{\prime}\right) d y \\
& \geq \int_{0}^{\infty} g(y) \mathcal{G}(y) \varphi\left(\frac{\varrho}{\mathcal{G}(y)}\right) d y \\
& \geq \varrho \int_{0}^{\infty} g(y) \ln \left(\frac{\varrho}{\mathcal{G}(y)}\right) d y \\
& \geq \varrho \ln \varrho G(0)+\varrho \int_{0}^{\infty} \partial_{y} G(y) \ln \mathcal{G}(y) d y \\
& \geq \varrho \int_{0}^{\infty} \frac{|G(y)|^{2}}{\mathcal{G}(y)} d y,
\end{aligned}
$$

whence (24) follows.

Proof of Proposition 7. We have

$$
\frac{d}{d t} H_{1}\left(g \mid g_{\varrho}\right)=\int_{0}^{\infty} y \ln g \partial_{t} g d y+\frac{2}{\varrho} \frac{d M_{2}(g)}{d t} .
$$

On the one hand, by (10), we have

$$
I:=\int_{0}^{\infty} y \ln g \partial_{t} g d y
$$




$$
\begin{aligned}
= & \int_{0}^{\infty} y^{2} \ln g(y) \partial_{y} g(y) d y+2 \int_{0}^{\infty} y \ln g(y) g(y) d y \\
& +\int_{0}^{\infty} \int_{0}^{\infty} y \ln \left(\frac{g\left(y+y^{\prime}\right)}{g(y)}\right) g(y) g\left(y^{\prime}\right) d y d y^{\prime} \\
= & -\int_{0}^{\infty} y^{2} \partial_{y} g(y) d y \\
& +\int_{0}^{\infty} \int_{0}^{\infty} y \ln \left(\frac{g\left(y+y^{\prime}\right)}{g(y)}\right) g(y) g\left(y^{\prime}\right) d y d y^{\prime} \\
= & 2 \varrho+\int_{0}^{\infty} \int_{0}^{\infty} y \ln \left(\frac{g\left(y+y^{\prime}\right)}{g(y)}\right) g(y) g\left(y^{\prime}\right) d y d y^{\prime} .
\end{aligned}
$$

On the other hand, (19) also reads

$$
\frac{2}{\varrho} \frac{d M_{2}(g)}{d t}=-\frac{2}{\varrho} M_{2}(g)+2 \varrho .
$$

Consequently,

$$
-\frac{d}{d t} H_{1}\left(g \mid g_{\varrho}\right)=-4 \varrho+\frac{2}{\varrho} M_{2}(g)+\int_{0}^{\infty} \int_{0}^{\infty} y^{\prime} g(y) g\left(y^{\prime}\right) \ln \left(\frac{g\left(y^{\prime}\right)}{g\left(y+y^{\prime}\right)}\right) d y^{\prime} d y .
$$

Next, a direct computation and (18) give

$$
M_{2}(g)=2 M_{1}(G)=2 M_{0}(\mathcal{G}) \text { and } \varrho=M_{1}(g)=M_{0}(G)=M_{0}\left(-\partial_{y} \mathcal{G}\right) .
$$

We then deduce from Lemma 8 and (25) that

$$
\begin{aligned}
-\frac{d}{d t} H_{1}\left(g \mid g_{\varrho}\right) & \geq 4 \int_{0}^{\infty} \partial_{y} \mathcal{G}(y) d y+\varrho \int_{0}^{\infty} \frac{\left|\partial_{y} \mathcal{G}(y)\right|^{2}}{\mathcal{G}(y)} d y+\frac{4}{\varrho} \int_{0}^{\infty} \mathcal{G}(y) d y \\
& =D_{1}(g)
\end{aligned}
$$

and the proof of Proposition 7 is complete.

Proposition 9 For $t_{2} \geq t_{1} \geq 0$, we have

$$
H_{0}\left(G\left(t_{2}\right) \mid G_{\varrho}\right)+\frac{1}{2} \int_{t_{1}}^{t_{2}} D_{1}(g(s)) d s \leq H_{0}\left(G\left(t_{1}\right) \mid G_{\varrho}\right),
$$

where $D_{1}(g)$ is still defined by (23).

The proof of Proposition 9 relies on the following lemma, which is again a modification of $[1$, Proposition 4.3].

Lemma 10 We have

$$
\int_{0}^{\infty} \int_{0}^{\infty} g(y) G\left(y^{\prime}\right) \ln \left(\frac{G\left(y^{\prime}\right)}{G\left(y+y^{\prime}\right)}\right) d y^{\prime} d y \geq \varrho \int_{0}^{\infty} \frac{|G(y)|^{2}}{\mathcal{G}(y)} d y .
$$


Proof of Lemma 10. Since $\varphi(r)=r \ln r, r \geq 0$, is convex, the Jensen inequality implies that

$$
\begin{aligned}
I & :=\int_{0}^{\infty} \int_{0}^{\infty} g(y) G\left(y^{\prime}\right) \ln \left(\frac{G\left(y^{\prime}\right)}{G\left(y+y^{\prime}\right)}\right) d y^{\prime} d y \\
& =\int_{0}^{\infty} g(y) \mathcal{G}(y) \int_{0}^{\infty} \varphi\left(\frac{G\left(y^{\prime}\right)}{G\left(y+y^{\prime}\right)}\right) \frac{G\left(y+y^{\prime}\right)}{\mathcal{G}(y)} d y^{\prime} d y \\
& \geq \int_{0}^{\infty} g(y) \mathcal{G}(y) \varphi\left(\int_{0}^{\infty} \frac{G\left(y^{\prime}\right)}{G\left(y+y^{\prime}\right)} \frac{G\left(y+y^{\prime}\right)}{\mathcal{G}(y)} d y^{\prime}\right) d y \\
& \geq \int_{0}^{\infty} g(y) \mathcal{G}(y) \varphi\left(\frac{\varrho}{\mathcal{G}(y)}\right) d y \\
& \geq \varrho \int_{0}^{\infty} g(y) \ln \left(\frac{\varrho}{\mathcal{G}(y)}\right) d y \\
& \geq \varrho \ln \varrho G(0)+\varrho \int_{0}^{\infty} \partial_{y} G(y) \ln \mathcal{G}(y) d y \\
& \geq \varrho \int_{0}^{\infty} \frac{|G(y)|^{2}}{\mathcal{G}(y)} d y,
\end{aligned}
$$

whence (27).

Proof of Proposition 9. We first notice that, by (10), $G$ satisfies

$$
\partial_{t} G(t, y)=y \partial_{y} G(t, y)+\left(1-\frac{G(t, 0)}{2}\right) G(t, y)+\frac{1}{2} \int_{0}^{y} g\left(t, y^{\prime}\right) G\left(t, y-y^{\prime}\right) d y^{\prime}
$$

for $(t, y) \in \mathbb{R}_{+}^{2}$. To obtain (28), we have used that

$$
\begin{aligned}
& \frac{1}{2} \int_{y}^{\infty} \int_{0}^{y^{\prime}} g\left(y^{\prime}-y^{\prime \prime}\right) g\left(y^{\prime \prime}\right) d y^{\prime \prime} d y^{\prime} \\
= & \frac{1}{2} \int_{y}^{\infty} \int_{0}^{y} g\left(y^{\prime}-y^{\prime \prime}\right) g\left(y^{\prime \prime}\right) d y^{\prime \prime} d y^{\prime}+\frac{1}{2} \int_{y}^{\infty} \int_{y}^{y^{\prime}} g\left(y^{\prime}-y^{\prime \prime}\right) g\left(y^{\prime \prime}\right) d y^{\prime \prime} d y^{\prime} \\
= & \frac{1}{2} \int_{0}^{y} g\left(y^{\prime \prime}\right) \int_{y}^{\infty} g\left(y^{\prime}-y^{\prime \prime}\right) d y^{\prime} d y^{\prime \prime}+\frac{1}{2} \int_{y}^{\infty} \int_{y^{\prime \prime}}^{\infty} g\left(y^{\prime}-y^{\prime \prime}\right) g\left(y^{\prime \prime}\right) d y^{\prime} d y^{\prime \prime} \\
= & \frac{1}{2} \int_{0}^{y} g\left(y^{\prime \prime}\right) G\left(y-y^{\prime \prime}\right) d y^{\prime \prime}+\frac{1}{2} \int_{y}^{\infty} g\left(y^{\prime \prime}\right) \int_{0}^{\infty} g\left(y^{\prime}\right) d y^{\prime} d y^{\prime \prime} \\
= & \frac{1}{2} \int_{0}^{y} g\left(y^{\prime}\right) G\left(y-y^{\prime}\right) d y^{\prime}+\frac{1}{2} G(0) G(y) .
\end{aligned}
$$

We infer from (19), (25) and (28) that

$$
\frac{d}{d t} H_{0}\left(G \mid G_{\varrho}\right)=\int_{0}^{\infty} \ln G \partial_{t} G d y+\frac{2}{\varrho} \frac{d M_{1}(G)}{d t}
$$




$$
\begin{aligned}
= & M_{0}(G)-\frac{M_{0}(g)}{2} \int_{0}^{\infty} G \ln G d y \\
& +\frac{1}{2} \int_{0}^{\infty} \int_{0}^{\infty} g(y) G\left(y^{\prime}\right) \ln \left(G\left(y+y^{\prime}\right)\right) d y d y^{\prime}-\frac{2 M_{1}(G)}{\varrho}+\varrho \\
= & 2 \varrho-\frac{2 M_{1}(G)}{\varrho}-\frac{1}{2} \int_{0}^{\infty} \int_{0}^{\infty} g(y) G\left(y^{\prime}\right) \ln \left(\frac{G\left(y^{\prime}\right)}{G\left(y+y^{\prime}\right)}\right) d y^{\prime} d y
\end{aligned}
$$

Thanks to Lemma 10 and the equality $M_{1}(G)=M_{0}(\mathcal{G})$, we obtain that

$$
-\frac{d}{d t} H_{0}\left(G \mid G_{\varrho}\right) \geq \frac{2 M_{0}(\mathcal{G})}{\varrho}-2 \varrho+\frac{\varrho}{2} \int_{0}^{\infty} \frac{\left|\partial_{y} \mathcal{G}(y)\right|^{2}}{\mathcal{G}(y)} d y .
$$

Noticing that

$$
\int_{0}^{\infty} \mathcal{G}(y) \partial_{y} \ln \mathcal{G}(y)=-\mathcal{G}(0)=-\varrho
$$

by (18), we end up with

$$
\begin{aligned}
-\frac{d}{d t} H_{0}\left(G \mid G_{\varrho}\right) \geq & \frac{2}{\varrho}\left\{\int_{0}^{\infty} \mathcal{G}(y) d y+\varrho \int_{0}^{\infty} \mathcal{G}(y) \partial_{y} \ln \mathcal{G}(y) d y\right. \\
& \left.+\frac{\varrho^{2}}{4} \int_{0}^{\infty} \mathcal{G}(y)\left|\partial_{y} \ln \mathcal{G}(y)\right|^{2} d y\right\} \\
\geq & \frac{2}{\varrho} \int_{0}^{\infty} \mathcal{G}(y)\left(1+\frac{\varrho}{2} \partial_{y} \ln \mathcal{G}(y)\right)^{2} d y \\
= & \frac{1}{2} D_{1}(g),
\end{aligned}
$$

which completes the proof of Proposition 9.

Remark 11 It follows from the Jensen inequality that

$$
H_{0}\left(G \mid G_{\varrho}\right) \leq H_{1}\left(g \mid g_{\varrho}\right) .
$$

Indeed, if $y \in \mathbb{R}_{+}$and $\varphi(r)=r \ln r, r \geq 0$, the Jensen inequality yields that

$$
\begin{aligned}
\int_{y}^{\infty} g\left(y^{\prime}\right) \ln \left(\frac{g\left(y^{\prime}\right)}{g_{\varrho}\left(y^{\prime}\right)}\right) d y^{\prime} & =\int_{y}^{\infty} \varphi\left(\frac{g\left(y^{\prime}\right)}{g_{\varrho}\left(y^{\prime}\right)}\right) g_{\varrho}\left(y^{\prime}\right) \frac{d y^{\prime}}{G_{\varrho}(y)} G_{\varrho}(y) \\
& \geq G_{\varrho}(y) \varphi\left(\int_{y}^{\infty} \frac{g\left(y^{\prime}\right)}{g_{\varrho}\left(y^{\prime}\right)} g_{\varrho}\left(y^{\prime}\right) \frac{d y^{\prime}}{G_{\varrho}(y)}\right) \\
& =G(y) \ln \left(\frac{G(y)}{G_{\varrho}(y)}\right),
\end{aligned}
$$

and the claim follows after integration over $\mathbb{R}_{+}$with respect to $y$. 
Proposition 12 For $t_{2} \geq t_{1} \geq 0$, we have

$$
L_{1}\left(G\left(t_{2}\right) \mid G_{\varrho}\right)+\int_{t_{1}}^{t_{2}} D_{L}(g(s)) d s=L_{1}\left(G\left(t_{1}\right) \mid G_{\varrho}\right)
$$

where

$$
D_{L}(g):=\frac{1}{2} \int_{0}^{\infty} \int_{0}^{\infty}\left(g_{\varrho}\left(y^{\prime}\right)+g\left(y^{\prime}\right)\right)\left[1-\operatorname{sign}\left(w\left(y+y^{\prime}\right) w(y)\right)\right]|w(y)| d y d y^{\prime} \geq 0,
$$

and $w=G-G_{\varrho}$.

Proof of Proposition 12. Since $G_{\varrho}$ is a stationary solution to $(28)$ and satisfies $G_{\varrho}(0)=2$, we have

$$
y \partial_{y} G_{\varrho}(y)+\frac{1}{2} \int_{0}^{y} g_{\varrho}\left(y^{\prime}\right) G_{\varrho}\left(y-y^{\prime}\right) d y^{\prime}=0 .
$$

Substracting this identity from (28), we obtain

$$
\partial_{t} w(t, y)=y \partial_{y} w(t, y)+\left(1-\frac{G(t, 0)}{2}\right) G(t, y)+\frac{1}{2} \Sigma(t, y)
$$

with

$$
\Sigma(t, y)=\int_{0}^{y}\left[g\left(t, y^{\prime}\right) G\left(t, y-y^{\prime}\right)-g_{\varrho}\left(y^{\prime}\right) G_{\varrho}\left(y-y^{\prime}\right)\right] d y^{\prime} .
$$

Now, using an integration by parts, we have

$$
\begin{aligned}
\int_{0}^{y}\left(g+g_{\varrho}\right)\left(y^{\prime}\right) w\left(y-y^{\prime}\right) d y^{\prime}= & \int_{0}^{y} g\left(y^{\prime}\right) G\left(y-y^{\prime}\right) d y^{\prime}+\int_{0}^{y} G_{\varrho}\left(y-y^{\prime}\right) \partial_{y} G\left(y^{\prime}\right) d y^{\prime} \\
& +\int_{0}^{y} g_{\varrho}\left(y^{\prime}\right) G\left(y-y^{\prime}\right) d y^{\prime}-\int_{0}^{y} g_{\varrho}\left(y^{\prime}\right) G_{\varrho}\left(y-y^{\prime}\right) d y^{\prime} \\
= & \Sigma(y)+G_{\varrho}(0) G(y)-G_{\varrho}(y) G(0) \\
& +\int_{0}^{y} G\left(y^{\prime}\right)\left(\partial_{y} G_{\varrho}\right)\left(y-y^{\prime}\right) d y^{\prime}+\int_{0}^{y} g_{\varrho}\left(y^{\prime}\right) G\left(y-y^{\prime}\right) d y^{\prime} \\
= & \Sigma(y)+2 G(y)-G_{\varrho}(y) G(0) .
\end{aligned}
$$

Consequently, (30) becomes

$$
\partial_{t} w(t, y)=y \partial_{y} w(t, y)-\frac{G(t, 0)}{2} w(t, y)+\frac{1}{2} \int_{0}^{y}\left(g\left(t, y^{\prime}\right)+g_{\varrho}\left(y^{\prime}\right)\right) w\left(t, y-y^{\prime}\right) d y^{\prime}
$$

for $(t, y) \in \mathbb{R}_{+}^{2}$. We multiply the previous equation by $\operatorname{sign}(w(t, y))$ and integrate over $\mathbb{R}_{+}$. Since $M_{0}\left(g_{\varrho}\right)=2$, we use the Fubini theorem and a change of variables to obtain that

$$
\frac{d}{d t}\|w\|_{L^{1}}=-\left(1+\frac{G(t, 0)}{2}\right)\|w\|_{L^{1}}
$$




$$
\begin{aligned}
& +\frac{1}{2} \int_{0}^{\infty} \int_{0}^{\infty}\left(g+g_{\varrho}\right)\left(y^{\prime}\right) \operatorname{sign}\left(w\left(y+y^{\prime}\right)\right) w(y) d y^{\prime} d y \\
= & -\frac{1}{2} \int_{0}^{\infty}\left(g_{\varrho}+g\right)\left(y^{\prime}\right) d y^{\prime}\|w\|_{L^{1}} \\
& +\frac{1}{2} \int_{0}^{\infty} \int_{0}^{\infty}\left(g+g_{\varrho}\right)\left(y^{\prime}\right) \operatorname{sign}\left(w\left(y+y^{\prime}\right)\right) w(y) d y^{\prime} d y \\
= & -D_{L}(g) .
\end{aligned}
$$

Proposition 12 then follows after integrating with respect to time.

Theorem 1 is now a straightforward consequence of Propositions 5, 7,9 and 12.

The end of this section is devoted to a description of an approximation procedure which allows us to rigorously justify the computations performed above. We first observe that solutions to (10), (11) enjoy the following properties.

Proposition 13 Let $f^{i n}$ and $\hat{f}^{i n}$ be two functions in $\mathcal{Y}_{p}$ and denote by $g$ and $\hat{g}$ the corresponding solutions to (10), (11). Then $g$ and $\hat{g}$ belong to $L^{\infty}\left(0, T ; L^{p}\left(\mathbb{R}_{+} ;(1+y) d y\right)\right)$ for each $T>0$ and there is a constant $C$ depending only on $\max \left\{M_{0}\left(f^{\text {in }}\right), M_{0}\left(\hat{f}^{\text {in }}\right)\right\}$ such that

$$
\|(g-\hat{g})(t)\|_{L^{1}} \leq\left\|f^{i n}-\hat{f}^{i n}\right\|_{L^{1}} e^{C t}, \quad t \geq 0 .
$$

In addition, if there are $\varepsilon>0$ and $C>0$ such that

$$
f^{i n}(y) \geq \varepsilon \exp \{-C(1+y)\}, \quad y \in \mathbb{R}_{+},
$$

then there exists $\gamma>0$ depending only on $M_{0}\left(f^{i n}\right)$ such that

$$
g(t, y) \geq \varepsilon \exp \left\{-\gamma(1+y) e^{t}\right\}, \quad(t, y) \in[0,+\infty) \times \mathbb{R}_{+} .
$$

Proof of Proposition 13. The fact that $g \in L^{\infty}\left(0, T ; L^{p}\left(\mathbb{R}_{+} ;(1+y) d y\right)\right)$ for each $T>0$ can be proved as [15, Corollary 3.2].

Concerning the continuous dependence (32), a formal proof is as follows: setting $w:=$ $g-\hat{g}$, we infer from (10) that

$$
\begin{aligned}
\frac{d}{d t}\|w\|_{L^{1}} & =\|w\|_{L^{1}}+\frac{1}{2} \int_{0}^{\infty} \operatorname{sign}(w(y)) \int_{0}^{y}(g+\hat{g})\left(y-y^{\prime}\right) w\left(y^{\prime}\right) d y^{\prime} d y \\
& -\frac{1}{2} \int_{0}^{\infty} \operatorname{sign}(w(y))\left\{\left(M_{0}(g)+M_{0}(\hat{g})\right) w+(g+\hat{g})(y)\left(M_{0}(g)-M_{0}(\hat{g})\right)\right\} d y \\
& \leq \frac{1}{2}\left(2-M_{0}(g)-M_{0}(\hat{g})\right)\|w\|_{L^{1}}+\frac{1}{2} \int_{0}^{\infty} \int_{0}^{\infty}(g+\hat{g})(y)\left|w\left(y^{\prime}\right)\right| d y^{\prime} d y \\
& +\frac{1}{2}\left(M_{0}(g)+M_{0}(\hat{g})\right)\|w\|_{L^{1}} \\
& \leq \frac{1}{2}\left(2+M_{0}(g)+M_{0}(\hat{g})\right)\|w\|_{L^{1}} .
\end{aligned}
$$


Since $M_{0}(g) \leq \max \left\{2, M_{0}\left(f^{\text {in }}\right)\right\}$ by (17), the inequality (32) follows.

We finally consider $f^{\text {in }}$ enjoying the positivity property $(33)$. For $(t, y) \in \mathbb{R}_{+}^{2}$, we put $a(t):=\gamma e^{t}$ and $\psi(t, y):=\varepsilon e^{-a(t)(1+y)}$, where

$$
\gamma:=\max \left\{C, \frac{2\left|M_{0}\left(f^{i n}\right)-2\right|}{\min \left\{2, M_{0}\left(f^{i n}\right)\right\}}\right\} .
$$

On the one hand, the non-negativity of $g$ and (10) ensure that

$$
\partial_{t} g \geq y \partial_{y} g+\left(2-M_{0}(g(t))\right) g .
$$

On the other hand, a straightforward computation, (17) and the choice of $\gamma$ imply that

$$
\partial_{t} \psi \leq y \partial_{y} \psi+\left(2-M_{0}(g(t))\right) \psi
$$

Since $g(0, y) \geq \psi(0, y)$ for $y \in \mathbb{R}_{+}$, the comparison principle entails that $g \geq \psi$.

Now, if $f^{\text {in }}$ is an arbitrary function in $\mathcal{Y}_{p}$, we introduce $f_{\varepsilon}^{\text {in }}(y)=f^{\text {in }}(y)+\varepsilon e^{-(1+y)}$ for $y \in \mathbb{R}_{+}$and $\varepsilon \in(0,1)$. Clearly, $\left(f_{\varepsilon}^{\text {in }}\right)$ is a bounded sequence in $\mathcal{Y}_{p}$ which converges toward $f^{i n}$. Denoting by $g_{\varepsilon}$ the solution to $(10),(11)$ with initial datum $f_{\varepsilon}^{\text {in }}$, we infer from Proposition 13 that $\left(g_{\varepsilon}(t)\right)$ converges to $g(t)$ in $L^{1}\left(\mathbb{R}_{+}\right)$for each $t \geq 0$. Also, arguing as in [15, Corollary 3.2], one can prove that $\left(g_{\varepsilon}(t)\right)$ is bounded in $L^{p}\left(\mathbb{R}_{+}\right)$for each $t \geq 0$. Consequently, we deduce from (19) and the Vitali theorem that

$$
\begin{aligned}
\left(H_{0}\left(g_{\varepsilon}(t) \mid g_{\varrho_{\varepsilon}}\right), H_{1}\left(g_{\varepsilon}(t) \mid g_{\varrho_{\varepsilon}}\right)\right) & \longrightarrow\left(H_{0}\left(g(t) \mid g_{\varrho}\right), H_{1}\left(g(t) \mid g_{\varrho}\right)\right), \\
\left(H_{0}\left(G_{\varepsilon}(t) \mid G_{\varrho_{\varepsilon}}\right), L_{1}\left(G_{\varepsilon}(t) \mid G_{\varrho_{\varepsilon}}\right)\right) & \longrightarrow\left(H_{0}\left(G(t) \mid G_{\varrho}\right), L_{1}\left(G(t) \mid G_{\varrho}\right)\right),
\end{aligned}
$$

with the obvious notation $\varrho_{\varepsilon}:=M_{1}\left(f_{\varepsilon}^{\text {in }}\right)$. Finally, since $g_{\varepsilon}(t)$ is bounded from below by a negative exponential by (34), the computations performed above can be rigorously justified for $g_{\varepsilon}(t)$ and Theorem 1 for $g$ follows from the previously established convergences.

\section{Convergence}

In this last section, we show how the information obtained in Theorem 1 allow us to prove the convergence stated in Theorem 2. Consider $f^{i n} \in \mathcal{Y}_{p}$ with $\varrho:=M_{1}\left(f^{i n}\right)$ and denote by $g$ the corresponding solution to $(10),(11)$. We also put

$$
G(t, y):=\int_{y}^{\infty} g\left(t, y^{\prime}\right) d y^{\prime}, \quad \mathcal{G}(t, y):=\int_{y}^{\infty} G\left(t, y^{\prime}\right) d y^{\prime}, \quad h(t, y)=\mathcal{G}(t, y)^{1 / 2}
$$

for $(t, y) \in \mathbb{R}_{+}^{2}$.

Lemma 14 Let $\left(t_{n}\right)_{n \geq 1}$ be an increasing sequence of positive real numbers such that $t_{n} \rightarrow$ $+\infty$. Introducing $g_{n}(t):=g\left(t+t_{n}\right), G_{n}(t):=G\left(t+t_{n}\right), \mathcal{G}_{n}(t):=\mathcal{G}\left(t+t_{n}\right)$ and $h_{n}(t):=$ 
$h\left(t+t_{n}\right)$ for $t \in[0,1]$ and $n \geq 1$, there are a subsequence of $\left(t_{n}\right)$ (not relabeled) and $g_{\infty} \in \mathcal{C}\left([0,1] ; L_{w}^{1}\left(\mathbb{R}_{+}\right)\right)$such that

$$
g_{n} \longrightarrow g_{\infty} \text { in } \mathcal{C}\left([0,1] ; L_{w}^{1}\left(\mathbb{R}_{+}\right)\right)
$$

and

$$
\left(G_{n}(t), \mathcal{G}_{n}(t), h_{n}(t)\right) \longrightarrow\left(G_{\infty}(t), \mathcal{G}_{\infty}(t), h_{\infty}(t)\right) \text { in } \mathcal{C}([0,+\infty))
$$

for each $t \in[0,1]$, where

$$
G_{\infty}(t, y):=\int_{y}^{\infty} g_{\infty}\left(t, y^{\prime}\right) d y^{\prime}, \quad \mathcal{G}_{\infty}(t, y):=\int_{y}^{\infty} G_{\infty}\left(t, y^{\prime}\right) d y^{\prime}, \quad h_{\infty}(t, y)=\mathcal{G}_{\infty}(t, y)^{1 / 2}
$$

for $(t, y) \in[0,1] \times \mathbb{R}_{+}$.

Here and below, $\mathcal{C}\left([0,1] ; L_{w}^{1}\left(\mathbb{R}_{+}\right)\right)$denote the space of continuous functions from $[0,1]$ in $L^{1}\left(\mathbb{R}_{+}\right)$endowed with its weak topology.

Proof of Lemma 14. It follows from (17), (19) and Proposition 5 that

$$
\{g(t), t \geq 0\} \text { is bounded in } \mathcal{Y}_{\ell},
$$

where $\mathcal{Y}_{\ell}$ is defined by (16). In particular, since $r \longmapsto r \ln r, r \geq 0$, is superlinear at infinity, we infer from (37) and the Dunford-Pettis theorem that $\left(g\left(t_{n}\right)\right)$ is weakly sequentially compact in $L_{1}^{1}\left(\mathbb{R}_{+}\right)$. In addition, (10) and (37) entail that

$$
t \longmapsto \int_{0}^{\infty} g_{n}(t, y) \psi(y) d y \quad \text { is bounded in } W^{1, \infty}(0,1)
$$

for any $\psi \in W^{1, \infty}\left(\mathbb{R}_{+}\right)$. With the help of the Dunford-Pettis and Arzelà-Ascoli theorems, we may then argue as in [8, Section 3.2] to conclude that there are a subsequence of $\left(g_{n}\right)$ (not relabeled) and $g_{\infty} \in \mathcal{C}\left([0,1] ; L_{w}^{1}\left(\mathbb{R}_{+}\right)\right)$such that (35) holds true. Since $\left(M_{2}\left(g_{n}(t)\right)\right)$ is bounded by (37), it also follows from (18) and (35) that

$$
M_{1}\left(g_{\infty}(t)\right)=M_{1}\left(g_{n}(t)\right)=M_{1}\left(g\left(t_{n}\right)\right)=\varrho, \quad t \in[0,1] .
$$

We next claim that

$$
G_{n}(t) \longrightarrow G_{\infty}(t) \quad \text { in } \quad \mathcal{C}([0,+\infty))
$$

for each $t \in[0,1]$. Indeed, since $\mathbf{1}_{[y,+\infty)}$ belongs to $L^{\infty}\left(\mathbb{R}_{+}\right)$for each $y \geq 0$, it readily follows from $(35)$ that $\left(G_{n}(t, y)\right)$ converges towards $G_{\infty}(t, y)$ for any $(t, y) \in[0,1] \times[0,+\infty)$. In addition, (37) implies that there is a positive constant $C$ such that

$$
\sup _{t \in[0,1]} \sup _{n \geq 1} \int_{0}^{\infty} g_{n}(t, y)\left|\ln \left(g_{n}(t, y)\right)\right| d y \leq C .
$$


Consequently, if $t \in[0,1], y \geq y^{\prime} \geq 0$ and $M:=\max \left\{2,\left|y-y^{\prime}\right|^{-1 / 2}\right\}>1$, we have

$$
\begin{aligned}
\left|G_{n}(t, y)-G_{n}\left(t, y^{\prime}\right)\right| & \leq \int_{y^{\prime}}^{y}\left|g_{n}(t, z)\right| d z \\
& \leq M\left|y-y^{\prime}\right|+\int_{\left\{\left[y^{\prime}, y\right] \cap\left\{g_{n}(t) \geq M\right\}\right\}} \frac{g_{n}(t, z)\left|\ln \left(g_{n}(t, z)\right)\right|}{\ln M} d z \\
& \leq M\left|y-y^{\prime}\right|+\frac{C}{\ln M},
\end{aligned}
$$

which implies the equicontinuity of $\left(G_{n}(t)\right)$ with respect to $y$. Since $G_{n}(t, y) \leq \varrho y^{-1}$ by (18), we are in a position to apply the Arzelà-Ascoli theorem and conclude that (39) holds true. We next employ a similar argument for $\mathcal{G}_{n}$ to complete the proof of Lemma 14.

It remains now to identify $g_{\infty}$. On the one hand, we infer from $(22)$ that $D_{1}(g) \in$ $L^{1}(0,+\infty)$. Consequently,

$$
\lim _{n \rightarrow+\infty} \int_{0}^{1} D_{1}\left(g_{n}(t)\right) d t=0 .
$$

On the other hand, since $\partial_{y} h_{n}=\mathcal{G}_{n}^{-1 / 2} \partial_{y} \mathcal{G}_{n} / 2$, we observe that

$$
D_{1}\left(g_{n}(t)\right)=\frac{4}{\varrho} \int_{0}^{\infty}\left(h_{n}(t)+\varrho \partial_{y} h_{n}(t)\right)^{2} d y
$$

and (36) and a lower semicontinuity argument imply that

$$
\int_{0}^{1} D_{1}\left(g_{\infty}(t)\right) d t \leq \liminf _{n \rightarrow+\infty} \int_{0}^{1} D_{1}\left(g_{n}(t)\right) d t .
$$

Therefore, $D_{1}\left(g_{\infty}(t)\right)=0$ for a.e. $t \in(0,1)$, that is, $h_{\infty}(t, y)+\varrho \partial_{y} h_{\infty}(t, y)=0$ a.e. in $(0,1) \times \mathbb{R}_{+}$. Thus, there is $\gamma(t)$ such that $h_{\infty}(t, y)=\gamma(t) e^{-y / \varrho}$. Recalling $(38)$ and the relationship between $h_{\infty}$ and $g_{\infty}$, we are led to $g_{\infty}(t)=g_{\varrho}$ for a.e. $t \in(0,1)$. Thanks to the weak continuity of $g_{\infty}$, this equality is actually valid for each $t \in[0,1]$.

Summarizing, we have proved that, for each increasing sequence $\left(t_{n}\right)$ of positive real numbers such that $t_{n} \rightarrow+\infty$, there is a subsequence $\left(t_{n_{k}}\right)$ of $\left(t_{n}\right)$ such that $\left(g\left(t_{n_{k}}\right)\right)$ converges weakly towards $g_{\varrho}$ in $L^{1}\left(\mathbb{R}_{+}\right)$. Since $\{g(t), t \geq 0\}$ is weakly sequentially compact in $L_{1}^{1}\left(\mathbb{R}_{+}\right)$ by (37) and has only one possible cluster point $g_{\varrho}$, Theorem 2 follows by a classical argument.

Acknowledgements. Partial support from the European Research Training Network HYKE HPRN-CT-2002-00282 is gratefully acknowledged.

\section{References}

[1] M. Aizenman and T.A. Bak, Convergence to equilibrium in a system of reacting polymers, Comm. Math. Phys. 65 (1979), 203-230. 
[2] D.J. Aldous, Deterministic and stochastic models for coalescence (aggregation, coagulation) : a review of the mean-field theory for probabilists, Bernoulli 5 (1999), 3-48.

[3] J. Bertoin, Eternal solutions to Smoluchowski's coagulation equation with additive kernel and their probabilistic interpretations, Ann. Appl. Probab. 12 (2002), 547-564.

[4] F.P. da Costa, On the dynamic scaling behaviour of solutions to the discrete Smoluchowski equations, Proc. Edinburgh Math. Soc. (2) 39 (1996), 547-559.

[5] M. Deaconu and E. Tanré, Smoluchowski's coagulation equation: probabilistic interpretation of solutions for constant, additive and multiplicative kernels, Ann. Scuola Norm. Sup. Pisa Cl. Sci. (4) 29 (2000), 549-579.

[6] P.G.J. van Dongen and M.H. Ernst, Scaling solutions of Smoluchowski's coagulation equation, J. Statist. Phys. 50 (1988), 295-329.

[7] R.L. Drake, A general mathematical survey of the coagulation equation, in Topics in Current Aerosol Research (part 2), International Reviews in Aerosol Physics and Chemistry, Pergamon Press, Oxford, 1972, pp. 203-376.

[8] M. Escobedo, Ph. Laurençot, S. Mischler and B. Perthame, Gelation and mass conservation in coagulation-fragmentation models, J. Differential Equations 195 (2003), 143-174.

[9] M. Escobedo, S. Mischler and M. Rodriguez Ricard, On self-similarity and stationary problem for fragmentation and coagulation models, (submitted).

[10] F. Filbet and Ph. Laurençot, Numerical simulation of the Smoluchowski coagulation equation, SIAM J. Sci. Comput. 25 (2004), 2004-2028.

[11] N. Fournier and Ph. Laurençot, Existence of self-similar solutions to Smoluchowski's coagulation equation, (submitted).

[12] S.K. Friedlander and C.S. Wang, The self-preserving particle size distribution for coagulation by brownian motion J. Colloid Interface Sci. 22 (1966), 126-132.

[13] M. Kreer and O. Penrose, Proof of dynamical scaling in Smoluchowski's coagulation equation with constant kernel, J. Statist. Phys. 75 (1994), 389-407.

[14] D.S. Krivitsky, Numerical solution of the Smoluchowski kinetic equation and asymptotics of the distribution function, J. Phys. A 28 (1995), 2025-2039.

[15] Ph. Laurençot and S. Mischler, The continuous coagulation-fragmentation equation with diffusion, Arch. Rational Mech. Anal. 162 (2002), 45-99.

[16] Ph. Laurençot and S. Mischler, Convergence to equilibrium for the continuous coagulation-fragmentation equation, Bull. Sci. Math. 127 (2003), 179-190.

[17] Ph. Laurençot and S. Mischler, On coalescence equations and related models, in "Modeling and computational methods for kinetic equations", P. Degond, L. Pareschi, G. Russo (eds.), Birkhäuser, Boston, 2004, pp. 321-356.

[18] M.H. Lee, A survey of numerical solutions to the coagulation equation, J. Phys. A 34, 10219-10241.

[19] F. Leyvraz, Scaling theory and exactly solved models in the kinetics of irreversible aggregation, Phys. Rep. 383 (2003), 95-212.

[20] A. Meesters and M.H. Ernst, Numerical evaluation of self-preserving spectra in Smoluchowski's coagulation theory, J. Colloid Interface Sci. 119 (1987), 576-587.

[21] G. Menon and R.L. Pego, Dynamical scaling in Smoluchowski's coagulation equations: uniform convergence, Preprint (2003). 
[22] G. Menon and R.L. Pego, Approach to self-similarity in Smoluchowski's coagulation equations, Comm. Pure Appl. Math. 57 (2004), 1197-1232.

[23] M. Smoluchowski, Drei Vorträge über Diffusion, Brownsche Molekularbewegung und Koagulation von Kolloidteilchen, Physik. Zeitschr. 17 (1916), 557-599.

[24] M. Smoluchowski, Versuch einer mathematischen Theorie der Koagulationskinetik kolloider Lösungen, Zeitschrift f. physik. Chemie 92 (1917), 129-168. 